\title{
Radical nephrectomy with resection of vena cava thrombus using extracorporeal circulation and deep hypothermic circulatory arrest
}

\author{
Gregory J. Nason ${ }^{1}$; Khaled Ajib ${ }^{1}$; Guan Hee Tan ${ }^{1}$; Dixon T.S. Woon ${ }^{1}$; George T. Christakis ${ }^{2}$; \\ Robert K. Nam ${ }^{1}$ \\ ${ }^{1}$ Division of Urology, Department of Surgery, Sunnybrook Health Sciences Centre, University of Toronto, ON, \\ Canada; ${ }^{2}$ Division of Cardiac Surgery, Department of Surgery, Sunnybrook Health Sciences Centre, University \\ of Toronto, ON, Canada
}

Cite as: Nason GJ, Ajib K, Tan GH, et al. Radical nephrectomy with resection of vena cava thrombus using extracorporeal circulation and deep hypothermic circulatory arrest. Can Urol Assoc J 2020 September 28; Epub ahead of print. http://dx.doi.org/10.5489/cuaj.6736

Published online September 28, 2020

$* * *$

\section{Abstract}

Introduction: Patients with renal cell carcinoma (RCC) with level 3 or 4 caval thrombus have a poor prognosis, with reported five-year survival rates of $30-40 \%$. The aim of this study was to assess the perioperative morbidity and long-term oncological outcomes for radical nephrectomy with resection of vena cava thrombus using a combined surgical approach, including extracorporeal circulation and deep hypothermic circulatory arrest.

Methods: A retrospective review was performed of the institutional case log to identify all radical nephrectomies with caval thrombus performed from January 2006 to May 2020. Results: Twenty-five patients were identified with level 2 thrombus in one (4\%), level 3 thrombus in eight (32\%), and level 4 in 16 (64\%). The median followup was 20.6 months (range $0.2-133.3$ ). The median age at surgery was 68.4 years (range $44.2-85.5$ ). Twenty-one (84\%) patients were symptomatic at presentation. Six (24\%) patients had distant metastases at diagnosis. The median circulatory arrest time was 15 minutes (range 6-35). The 30-day grade $\geq 3$ complication rate was $8 \%$. The 30 -day mortality rate was $8 \%$. The one-year, twoyear, three-year, and five-year recurrence-free survival (RFS) rates were 53\%, 18\%, 10\%, and $10 \%$, respectively. The median time to systemic treatment was 7.7 months (range $1.2-$ 25.7). The one-year, two-year, three-year, five-year overall survival (OS) rates were $70 \%$, $43 \%, 36 \%$, and $31 \%$, respectively.

Conclusions: Radical nephrectomy with resection of vena cava thrombus using extracorporeal circulation and deep hypothermic circulatory arrest is associated with some morbidity and mortality but remains a safe and effective strategy for advanced RCC patients who would otherwise be managed palliatively. 


\section{Introduction}

Renal cell carcinoma (RCC) is one of the most common urological malignancies with $\sim 7000$ new kidney cancers diagnosed in Canada in 2019 [1]. With modern imaging, the majority are diagnosed incidentally [2] and are organ-confined at diagnosis [3]. The classic 'Beck's' triad of flank pain, visible hematuria and a palpable abdominal mass is now a rare presentation (6$10 \%$ ) and usually correlates with aggressive histology and advanced disease [4]. RCC extends into the inferior vena cava in $\sim 10 \%$ of cases at diagnosis and up to the right atrium in $1 \%$ of cases [5]. In these scenarios, patients often present acutely with symptoms such as pain, cardiovascular compromise due to the concomitant presence of locally advanced bulky tumour, vena cava involvement (+/- obstruction), and/or distant metastases [6].

Patients with advanced RCC with level 3 or 4 caval thrombus usually have a poor prognosis with reported 5 year survival rates of $30-40 \%$ but in a select cohort surgical resection may be beneficial, even for symptomatic relief [7-10]. Surgery with supradiaphragmatic thrombus requires thoracic access to the vena cava (+/- right atrium). A multidisciplinary approach involving cardiac surgeons is often required with extracorporeal circulation, and deep hypothermic circulatory arrest. These cases are a challenging undertaking with significant 30 -day morbidity rates of up to $40 \%$ and 30 -day mortality rates of $\sim 10-22 \%[6,8,9]$. However, there are little other potentially curative options available to these patients and without surgery they are managed palliatively. The median survival of untreated RCC with any tumor thrombus is $\sim 5$ months [11].

The aim of this study was to assess the perioperative morbidity and the long term oncological outcomes for radical nephrectomy with resection of vena cava thrombus using a combined surgical approach including extracorporeal circulation and deep hypothermic circulatory arrest.

\section{Methods}

A retrospective review was performed of the institutional case log to identify all radical nephrectomies performed by a single surgeon (RN) from January 2006 to May 2020. From this list, we identified cases with vena cava thrombus that required the involvement of the cardiac surgery team for cardiopulmonary bypass and hypothermic circulatory arrest. Patient demographics, operative details and pathological characteristics were collated from each patient's electronic chart, detailed in Table 1. Follow-up was determined from the date of surgery until date of last clinic follow-up or death.

All patients underwent preoperative evaluation to stage their disease and determine the proximal extent of vena cava thrombus. Assessment included routine laboratory and radiological investigations with chest xray, CT, Doppler ultrasonography and/or MRI. Patients were also evaluated in the anaesthetic pre-operative clinic to assess their perioperative risk.

Surgery was performed as a joint case with the cardiac surgery team usually in the cardiac OR to facilitate cardiopulmonary bypass and hypothermic circulatory arrest. Our surgical technique involved a Chevron incision for abdominal access. The kidney was fully 
mobilised in a standard fashion for a radical nephrectomy and ready for removal apart from the renal vein prior to commencing cooling or going on bypass. We aimed to ligate the renal artery early. The cardiac surgeons performed a separate median sternotomy and the pericardium opened. The right atrium, aorta, intrapericardial IVC, and right superior pulmonary vein were exposed. Patients were then systemically heparinized. The ascending aorta and right atrium were cannulated. A vent cannula was inserted in ascending aorta. Cooling was commenced and during this phase the IVC was exposed. The aorta was clamped, cardiac arrest was achieved by antegrade crystalloid cardioplegia. Patients were cooled to $18^{\circ} \mathrm{C}$. Once the circulation was arrested the IVC was opened to allow all visible tumour thrombus to be removed. Dissection was performed from above and below as required and the thrombus was removed en-bloc with the kidney specimen. The IVC was then repaired, circulation re-started and re-warming begun. Once off bypass any residual coagulopathy was corrected. It was not our practice to embolize the kidney pre-operatively. We also do not routinely biopsy large kidney masses like these as it is unlikely to change management and delays time to intervention.

Categorical variables such tumor location, thrombus level, presentation, recurrence and readmission rates were summarized with counts and percentages. Continuous variables such as age at diagnosis, tumor size, blood loss, circulatory arrest time, length of stay and follow-up will be summarized with median and range. The level of significance was set at $\mathrm{p}=0.05$. Statistical analyses were performed using version 9.4 of the SAS system for Windows (2002-2012 SAS Institute, Inc., Cary, NC).

The study was approved by institutional review board.

\section{Results}

During the study period, 518 radical nephrectomies were performed by a single surgeon. Of these, 25 (4.8\%) patients underwent radical nephrectomy with resection of vena cava thrombus using extracorporeal circulation and deep hypothermic circulatory arrest. The median follow up was 20.6 months $(0.2-133.3)$. The median age at surgery was 68.4 years (Range 44.2-85.5). 21 (84\%) patients were symptomatic at presentation with abdominal pain $(n=5,20 \%)$ and shortness of breath $(n=5,20 \%)$ being the most common presenting symptoms. The median size of tumor on cross sectional imaging at diagnosis was $9 \mathrm{~cm}$ (Range 5.6-14) with level 2 thrombus in 1 (4\%), level 3 in 8 (32\%) patients and level 4 in 16 $(64 \%)$ patients. $6(24 \%)$ patients had distant metastases at diagnosis. Patient demographics and preoperative characteristics are detailed in Table 1.

The median intra-operative blood loss was 2000 mls (Range 500-9000) with 21 (84\%) patients requiring a blood transfusion. The median circulatory arrest time was 15 mins (Range 6-35). Adjuvant procedures were performed in 9 (36\%) patients. The median length of stay was 12 days (Range 7-83). Clavien-Dindo complications $\geq 3$ occurred in 6 (24\%) patients, Table 2 . The 30 -day grade $\geq 3$ complication rate was $8 \%$ ( 2 patients- 2 dialysis temporarily for AKI). There were no intra-operative deaths. The 30-day mortality rate was $8 \%$ (2 patients). One was an 85 year old female with a history of Type 2 Diabetes Mellitus, 
Hypertension, Ischaemic heart disease, chronic kidney disease grade 3B (eGFR 39ml/min). American Society of Anesthesiologists (ASA) grade 4. She presented with functional decline and shortness of breath. CT showed a $11.5 \mathrm{~cm}$ left renal mass with a level 4 thrombus with oligometastatic disease to the bone. She required intraoperative blood transfusion but became severely overloaded. Post-operatively she was anuric and required iontropic support in ICU. She was commenced on continuous renal replacement therapy. Despite ICU management, she died within 24 hours in ICU. The second was an 85 year old lady with a history of hypertension, mitral regurgitation, congestive cardiac failure (CCF), newly diagnosed pulmonary embolus. ASA grade 4. She presented with shortness of breath, pain and leg swelling. CT showed a $5 \mathrm{~cm}$ right renal mass with a level 4 thrombus. She died post operative day 3 in ICU despite inotropic support from worsening CCF. Pathological characteristics are detailed in Table 3.

The 1-year, 2-year, 3-year, 5-year recurrence-free survival (RFS) rate were 53\%, $18 \%, 10 \%$, and 10\%, respectively, Figure 1. Disease recurrence occurred in $17(68 \%)$ patients at a median of 8.9 months (1.2-25.7). $16(64 \%)$ patients received adjuvant and/or salvage treatment. The median time to systemic treatment was 7.7 months (Range 1.2 -25.7). The 1year, 2-year, 3-year, 5-year overall survival (OS) rate were $70 \%, 43 \%, 36 \%$ and $31 \%$ respectively, Figure 2.

There was no difference in median RFS (22.1 months vs 18.1 months, $\mathrm{p}=0.36$ ) or median OS (20.6 months vs 18 months, $p=0.71$ ) based upon thrombus level 3 or 4 , Figure 3 and 4 . There was no difference in grade $\geq 3$ complications for level 3 or 4 thrombus, $(25 \%$ vs $25 \%, \mathrm{p}=1.0$ ). For the RCC patients (exclude 2 adrenocortical carcinomas), there was no difference in median OS based upon metastatic stage at diagnosis, (22.1 months vs 3.2 months, $\mathrm{p}=0.16$ ), Figure 5. Of the M0 RCC patients at diagnosis, there was no difference in median OS based upon the receipt of systemic treatment following nephrectomy, (34.5 months vs 22.1 months, $\mathrm{p}=0.31$ ), Figure 6 .

\section{Discussion}

Our study has demonstrated that radical nephrectomy with resection of vena cava thrombus using extracorporeal circulation and deep hypothermic circulatory arrest is a complex procedure associated with significant morbidity and mortality rates. The 30-day morbidity (Clavien-Dindo $\geq 3$ ) and mortality rates of $8 \%$ need to be clearly explained to any patient considering this procedure. The median survival of 20.6 months needs to be weighed against the perioperative risk. Despite these grave figures, an aggressive surgical resection is beneficial in a select group of patients who otherwise would be managed palliatively. The surgical aims of this procedure are threefold- debulking for symptom relief, prevention of metastases and prevention of fatal cardiovascular compromise such as heart failure and pulmonary embolus $[5,12]$. The natural history of this aggressive disease is demonstrated by a short interval to recurrence of only 8.9 months with only $10 \%$ of patients reaching 3 years without progression. Despite such a high recurrence/progression rate, with current systemic options, the 5-year OS remains $31 \%$ with a median survival of 20.6 months. By contrast, 
Haidar et al., reported the combined median survival for untreated (any level) caval thrombus was only 5 months [13]. Reese et al, demonstrated that the extent of thrombus and presence of metastases were predictors of mortality in an untreated population [11].

Given the complexity of the procedure, there is limited data within the literature. Schuh et al reported the largest series of level 4 thrombus in a multi-institutional study in 2011 [9]. They reported a higher per-operative mortality of $22 \%$ and a lower median survival of 15 months- however their series was restricted to level 4 thrombus where as a third of our cases were level 3. Interestingly, in our study the level of thrombus (3 vs 4) did not significantly affect perioperative complication rates or long-term RFS and OS rates. In comparison, Zastrow et al reported a series of 66 patients with level 1-4 thrombus. With a similar follow up to our series, they reported an improved median OS of 47 months over twice as long as the median survival in our series. The likely explanation being that less than half their series had level 3 or 4 thrombus. Surprisingly in that study, level of thrombus was not a predictor of survival on multivariable analysis [14]. Level of thrombus has been shown to be a predictor of survival in other studies but mainly when comparing supra- (3/4) and infra- (1/2) diaphragmatic levels [15-17].

The question regarding nephrectomy in the setting of metastatic disease has been debated in recent years. The CARMENA trial demonstrated that systemic treatment alone in the form of Sunitinib was not inferior to cytoreductive nephrectomy followed by Sunitinib for intermediate or poor risk metastatic RCC [18]. This trial was criticised however for slow accrual, incomplete accrual and a heavy weighting towards poor risk patients. There is an argument that there remains a role in the well selected patient [19]. The CARMENA trial also failed to report on symptoms and rates of thrombus making it difficult to apply the results to a symptomatic metastatic patient with a level 3 or 4 thrombus. Although our results did not reach statistical significance for a difference in survival based upon metastatic stage at present, there is a clear divergence in the survival curves in Figure 5 and likely just limited by our low numbers. Another concern regarding the use of cytoreductive nephrectomy is whether patients will recover sufficiently to receive systemic treatment. Palumbo et al, reported older ( $>71 \mathrm{yrs}$ ) frail patients were more likely to experience higher rates of overall complications, failure to recover and in-hospital mortality following cytoreductive nephrectomy [20]. Figure 6 in our study again demonstrates a divergence in the survival curves for those who received adjuvant therapy- we need to be cogniscent of frail older patients and if they will recover sufficiently for systemic treatment.

The role for centralisation of complex multi-disciplinary surgeries is often discussed. Radical nephrectomy with caval thrombectomy centralizes itself to some extent given many hospitals do not have the expertise on site to facilitate extracorporeal circulation and deep hypothermic circulatory arrest. Toren et al., reported a national population cohort of radical nephrectomy and thrombectomy in 2013 [21]. Patients who underwent concomitant cardiac bypass had increased in-hospital mortality and complications. In an Ontario based review, Yap et al, called for regionalization given was a predictor of early mortality in thrombectomy 
cases [22]. Clearly complex surgery of this nature should be directed towards high volume surgeons with appropriate multi-disciplinary support.

Our study is limited by its retrospective non-comparative nature and small numbers which has likely resulted in some of our survival analyses failing to reach significance despite clear trends in the Kaplan Meier curves. Furthermore our study lacks data regarding symptom improvement following surgery. Despite these facts we report a relatively large Canadian experience of this complex surgery which delineates the morbidity and mortality rates important for an appropriately consented patient.

\section{Conclusions}

Radical nephrectomy with resection of vena cava thrombus using extracorporeal circulation and deep hypothermic circulatory arrest is associated with some morbidity and mortality but remains a safe and effective strategy for advanced RCC patients who would otherwise be managed palliatively. 


\section{References}

1. https://www.cancer.ca/en/cancer-information/cancer-type/kidney/statistics (Accessed 13th May 2020)

2. Ljungberg $\mathrm{B}$, Bensalah $\mathrm{K}$, Canfield $\mathrm{S}$, et al. EAU guidelines on renal cell carcinoma: 2014 update. Eur Urol. 2015;67(5):913-924.

3. Nason GJ, McGuire BB, Kelly ME, et al. Clinico-pathological analysis of renal cell carcinoma demonstrates decreasing tumour grade over a 17-year period. Can Urol Assoc J. 2014 Mar;8(3-4):125-32.

4. Lee CT, Katz J, Fearn PA, et al. Mode of presentation of renal cell carcinoma provides prognostic information. Urol Oncol. 2002 Jul-Aug; 7(4):135-40

5. Blute ML, Leibovich BC, Lohse CM, et al. The Mayo Clinic experience with surgical management, complications and outcome for patients with renal cell carcinoma and venous tumour thrombus. BJU Int. 2004;94(1):33-41.

6. Nini A, Capitanio U, Larcher A, et al. Perioperative and Oncologic Outcomes of Nephrectomy and Caval Thrombectomy Using Extracorporeal Circulation and Deep Hypothermic Circulatory Arrest for Renal Cell Carcinoma Invading the Supradiaphragmatic Inferior Vena Cava and/or Right Atrium. Eur Urol. 2018;73(5):793-799.

7. Dashkevich A, Bagaev E, Hagl C, et al. Long-term outcomes after resection of Stage IV cavoatrial tumour extension using deep hypothermic circulatory arrest. Eur J Cardiothorac Surg. 2016;50(5):892-897.

8. Dominik J, Moravek P, Zacek P, et al. Long-term survival after radical surgery for renal cell carcinoma with tumour thrombus extension into the right atrium. BJU Int. 2013;111(3 Pt B):E59-E64.

9. Shuch B, Crispen PL, Leibovich BC, et al. Cardiopulmonary bypass and renal cell carcinoma with level IV tumour thrombus: can deep hypothermic circulatory arrest limit perioperative mortality? BJU Int. 2011;107(5):724-728.

10. Gagné-Loranger M, Lacombe L, Pouliot F, et al. Renal cell carcinoma with thrombus extending to the hepatic veins or right atrium: operative strategies based on 41 consecutive patients. Eur J Cardiothorac Surg. 2016;50(2):317-321.

11. Reese AC, Whitson JM, Meng MV. Natural history of untreated renal cell carcinoma with venous tumor thrombus. Urol Oncol. 2013;31(7):1305-1309.

12. Pagano F, Dal Bianco M, Artibani W, et al. Renal cell carcinoma with extension into the inferior vena cava: problems in diagnosis, staging and treatment. Eur Urol. 1992;22(3):200-3.

13. Haidar GM, Hicks TD, El-Sayed HF, et al. Treatment options and outcomes for caval thrombectomy and resection for renal cell carcinoma. J Vasc Surg Venous Lymphat Disord. 2017;5(3):430-436.

14. Zastrow S, Leike S, Oehlschläger S, et al. Surgery for renal cell cancer extending into the inferior vena cava - evaluation of survival and perioperative complications using a standardized classification system. BJU Int. 2011;108(9):1439-1443.

15. Klaver S, Joniau S, Suy R, et al. Analysis of renal cell carcinoma with subdiaphragmatic macroscopic venous invasion (T3b). BJU Int. 2008;101(4):444-449. 
16. Haferkamp A, Bastian PJ, Jakobi H, et al. Renal cell carcinoma with tumor thrombus extension into the vena cava: prospective long-term followup. J Urol. 2007;177(5):1703-1708.

17. Parekh DJ, Cookson MS, Chapman W, et al. Renal cell carcinoma with renal vein and inferior vena caval involvement: clinicopathological features, surgical techniques and outcomes. J Urol. 2005;173(6):1897-1902.

18. Méjean A, Ravaud A, Thezenas S, et al. Sunitinib Alone or after Nephrectomy in Metastatic Renal-Cell Carcinoma. N Engl J Med. 2018;379(5):417-427.

19. Motzer RJ, Russo P. Cytoreductive Nephrectomy - Patient Selection Is Key. N Engl J Med. 2018;379(5):481-482.

20. Palumbo C, Knipper S, Dzyuba-Negrean C, et al. Complication rates, failure to rescue and in-hospital mortality after cytoreductive nephrectomy in the older patients. $\mathrm{J}$ Geriatr Oncol. 2020;11(4):718-723.

21. Toren P, Abouassaly R, Timilshina N, et al. Results of a national population-based study of outcomes of surgery for renal tumors associated with inferior vena cava thrombus. Urology. 2013;82(3):572-577.

22. Yap SA, Horovitz D, Alibhai SM, et al. Predictors of early mortality after radical nephrectomy with renal vein or inferior vena cava thrombectomy - a population-based study. BJU Int. 2012;110(9):1283-1288.

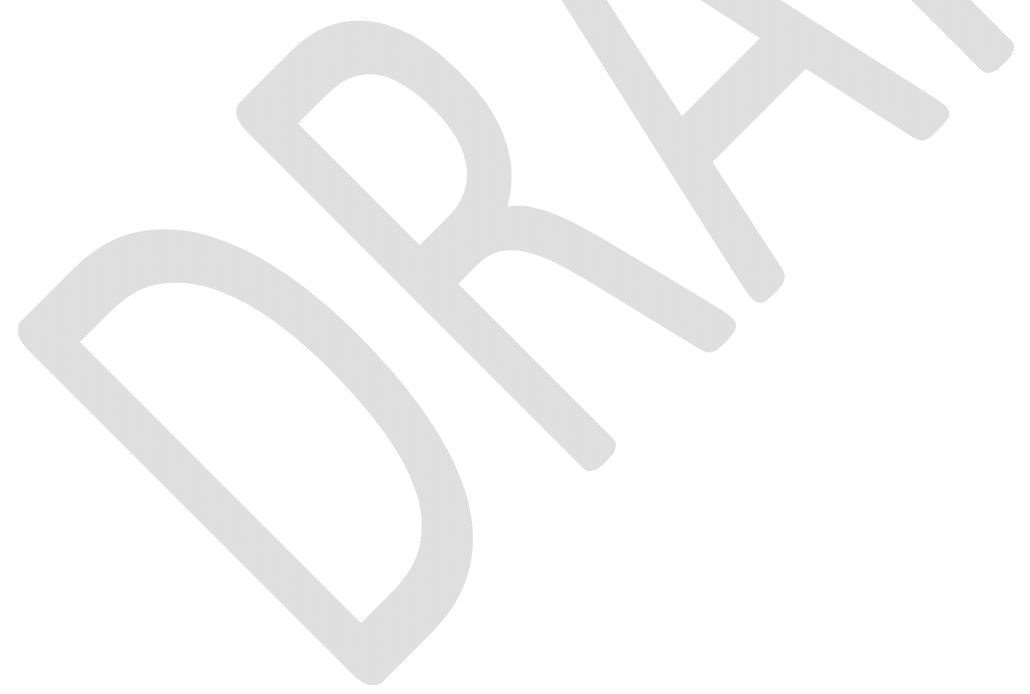




\section{Figures and Tables}

Fig. 1. Recurrence-free survival.

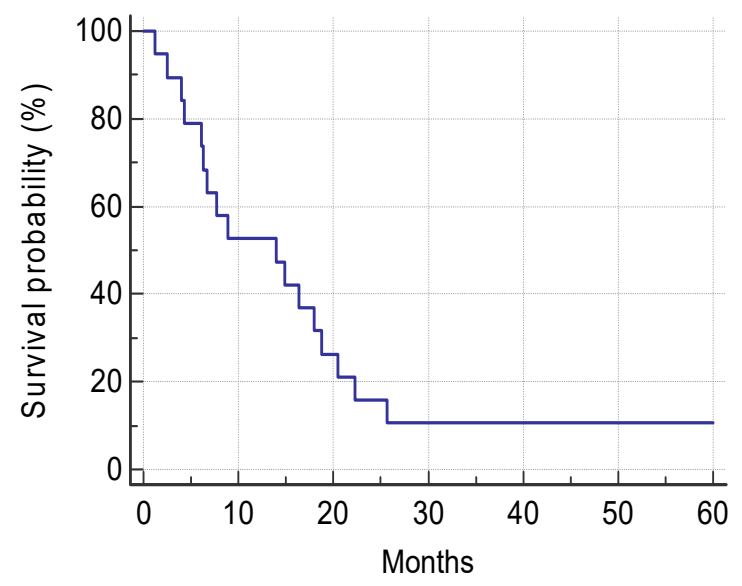

Fig. 2. Overall survival.

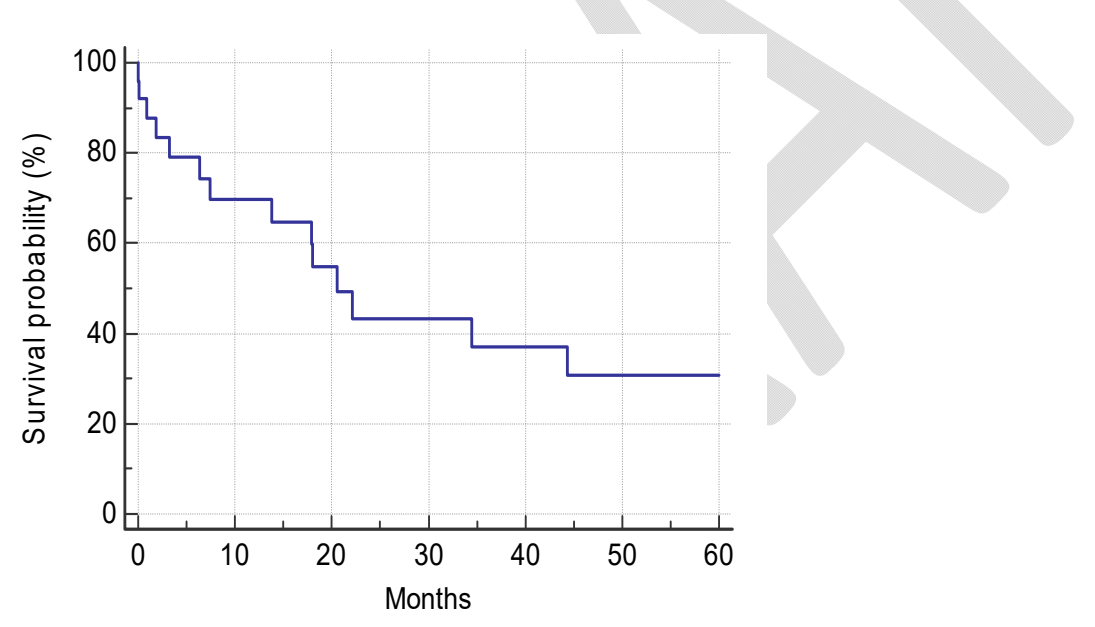


Fig. 3. Recurrence-free survival stratified by thrombus level.

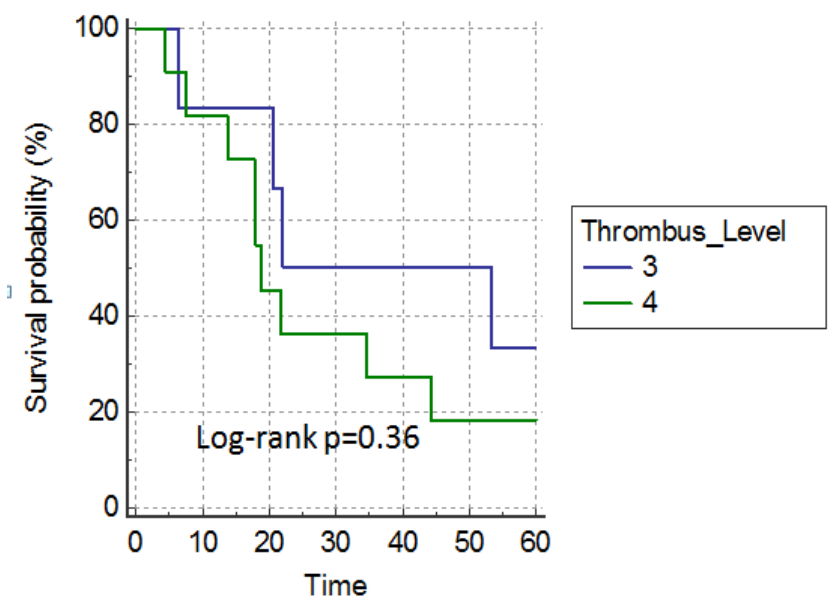

Fig. 4. Overall survival stratified by thrombus level.

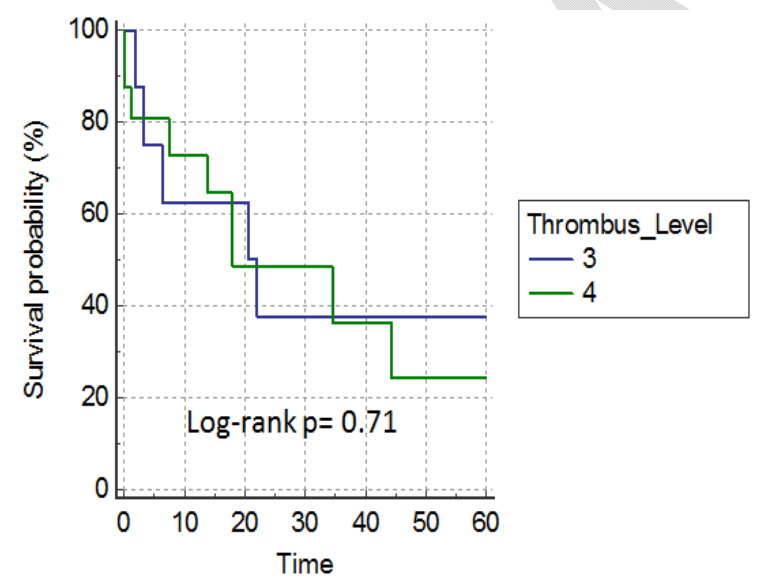


Fig. 5. Overall survival stratified by metastatic stage at diagnosis.

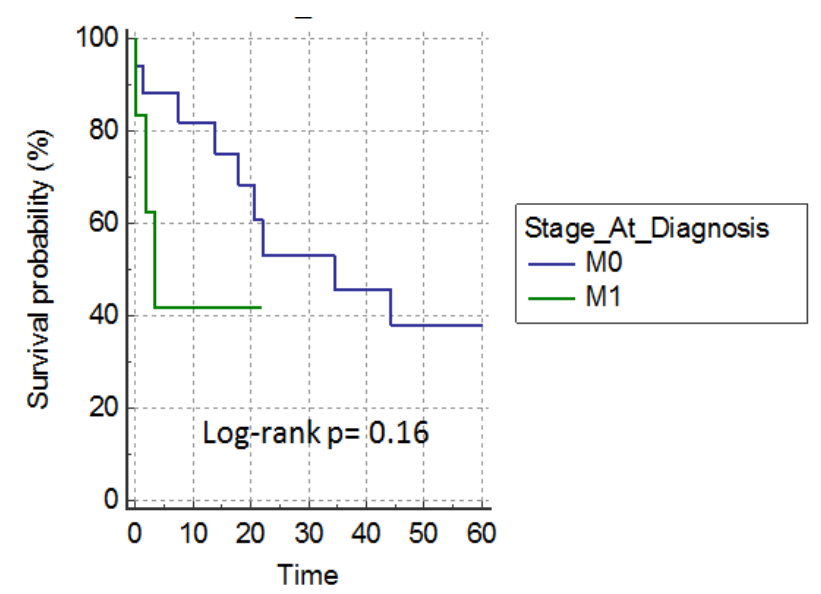

Fig. 6. Overall survival stratified by the receipt of systemic treatment after nephrectomy for M0 disease.

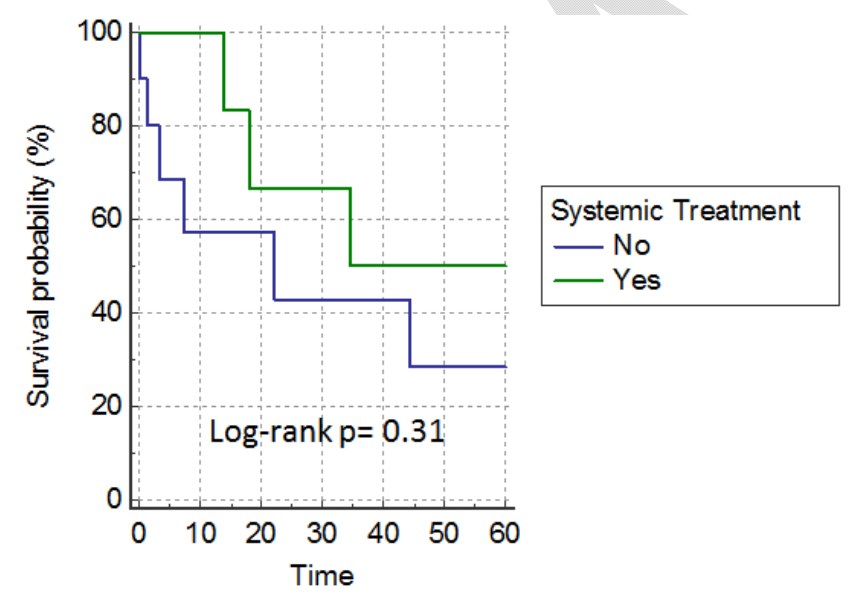




\begin{tabular}{|c|c|}
\hline Median age at surgery, years (range) & $68.4(44.2-85.5)$ \\
\hline \multicolumn{2}{|l|}{ Gender, $\mathrm{n}(\%)$} \\
\hline Male & $16(64)$ \\
\hline Female & $9(36)$ \\
\hline Smoker, n (\%) & $13(52)$ \\
\hline \multicolumn{2}{|l|}{ Laterality of tumor, $\mathrm{n}(\%)$} \\
\hline Right & $16(64)$ \\
\hline Left & $9(36)$ \\
\hline \multicolumn{2}{|l|}{ Location of tumor, n (\%) } \\
\hline Upper pole & $15(60)$ \\
\hline Lower pole & $10(40)$ \\
\hline Median size of tumor on imaging, $\mathrm{cm}$ (range) & $9(5.6-14)$ \\
\hline \multicolumn{2}{|l|}{ Thrombus level, $\mathrm{n}(\%)$} \\
\hline 2 (infrahepatic) & $1(4)$ \\
\hline 3 (suprahepatic) & $8(32)$ \\
\hline 4 (supra-diaphragmatic, including intra-atrial) & $16(64)$ \\
\hline \multicolumn{2}{|l|}{ Presentation, $\mathrm{n}(\%)$} \\
\hline Incidental & $4(16)$ \\
\hline Symptomatic & $21(84)$ \\
\hline \multicolumn{2}{|l|}{ Metastases at diagnosis } \\
\hline M0 & $19(76)$ \\
\hline M1 & $6(24)$ \\
\hline \multicolumn{2}{|l|}{ Primary presenting symptoms, $\mathrm{n}(\%)$} \\
\hline Hematuria & $4(16)$ \\
\hline Pain & $5(20)$ \\
\hline Leg swelling & $4(16)$ \\
\hline Lethargy & $1(4)$ \\
\hline Shortness of breath & $5(20)$ \\
\hline Weight loss & $2(8)$ \\
\hline Median preoperative hemoglobin, $\mathrm{g} / \mathrm{L}$ (range) & $102(74-161)$ \\
\hline Median preoperative creatinine, $\mu \mathrm{mol} / \mathrm{L}$ (range) & $120(66-285)$ \\
\hline
\end{tabular}




\begin{tabular}{|c|c|}
\hline Median blood loss, ml (range) & $2000(500-9000)$ \\
\hline Blood transfusions, $\mathrm{n}(\%)$ & $21(84)$ \\
\hline Median circulatory arrest time, mins (range) & $15(6-35)$ \\
\hline Adjuvant procedures, $\mathrm{n}(\%)$ & 9 patients $(36)$ \\
\hline Splenectomy & $3(12)$ \\
\hline Distal pancreatectomy & $3(12)$ \\
\hline Liver resection & $1(4)$ \\
\hline Duodenal repair & $2(8)$ \\
\hline Hemi colectomy & $1(4)$ \\
\hline Appendectomy & $1(4)$ \\
\hline Cholecystectomy & $1(4)$ \\
\hline IVC filter insertion & $1(4)$ \\
\hline Incision, $\mathrm{n}(\%)$ & +2 \\
\hline Chevron and sternotomy & $23(92)$ \\
\hline Midline and sternotomy & $2(8)$ \\
\hline Median length of stay, days (range) & $12(7-83)$ \\
\hline Clavien-Dindo complications, $\mathrm{n}(\%)$ & \\
\hline 1 & 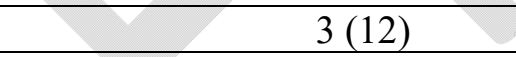 \\
\hline 2 & 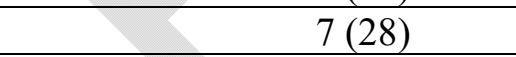 \\
\hline 3 & 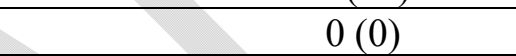 \\
\hline $\begin{array}{l}4 \\
\text { AKI requiring temporary dialysis } \\
\text { AKI requiring temporary dialysis }\end{array}$ & $2(8)$ \\
\hline 5 & $4(16)$ \\
\hline 30 -day grade $\geq 3, \mathrm{n}(\%)$ & $2(8)$ \\
\hline Intraoperative mortality, n (\%) & $0(0)$ \\
\hline 30-day mortality, n (\%) & $2(8)$ \\
\hline Readmissions, $\mathrm{n}(\%)$ & 5 \\
\hline 30-day & $3(12)$ \\
\hline 90-day & $1(4)$ \\
\hline 30-day ER presentation & $6(24)$ \\
\hline Recurrence, $\mathrm{n}(\%)$ & $17(68)$ \\
\hline Median time to recurrence, months (range) & $8.9(1.2-25.7)$ \\
\hline Adjuvant/salvage treatments, $\mathrm{n}(\%)$ & $16(64)$ \\
\hline Systemic therapy alone & $6(24)$ \\
\hline SBRT alone & $4(16)$ \\
\hline Systemic therapy and SBRT & $6(24)$ \\
\hline Median followup, months (range) & $20.6(0.2-133.3)$ \\
\hline
\end{tabular}

AKI: acute kidney injury; ER: emergency room; IVC: inferior vena cava; SBRT: stereotactic body radiotherapy. 


\begin{tabular}{|l|c|}
\hline Table 3. Histological details & \\
\hline Histological subtype, $\mathrm{n}(\%)$ & $18(72)$ \\
\hline Clear-cell & $4(16)$ \\
\hline Papillary (type 2) & $1(4)$ \\
\hline Translocation carcinoma & $2(8)$ \\
\hline Adrenocortical carcinoma & $0(0)$ \\
\hline Sarcomatoid features, n (\%) & $2(8)$ \\
\hline Rhabdoid features, $\mathrm{n}(\%)$ & \\
\hline Fuhrman grade & $0(0)$ \\
\hline 1 & $3(12)$ \\
\hline 2 & $10(40)$ \\
\hline 3 & $9(36)$ \\
\hline 4 & $3(12)$ \\
\hline N/A & $9(5.6-19)$ \\
\hline Median pathological tumor size, cm (range) & $6(24)$ \\
\hline Positive surgical margin, $\mathrm{n}(\%)$ & $21(84)$ \\
\hline Pathological stage & $4(16)$ \\
\hline pT3 & \\
\hline pT4 & \\
\hline
\end{tabular}

